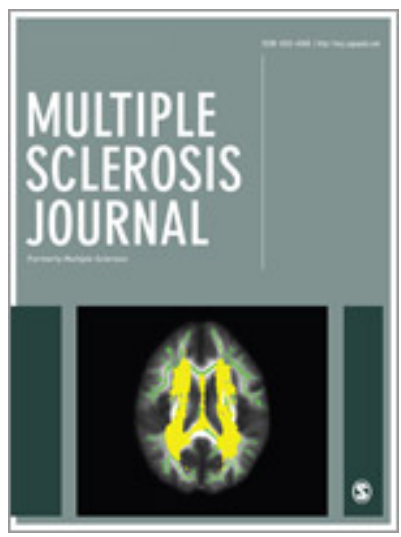

\title{
Risk of cancer among multiple sclerosis patients, siblings and population controls: a prospective cohort study
}

\begin{tabular}{|c|c|}
\hline Journal: & Multiple Sclerosis Journal \\
\hline Manuscript ID & MSJ-19-0363.R1 \\
\hline Manuscript Type: & Original Research Paper \\
\hline $\begin{array}{r}\text { Date Submitted by the } \\
\text { Author: }\end{array}$ & 03-Jul-2019 \\
\hline Complete List of Authors: & $\begin{array}{l}\text { Grytten, Nina; Norwegian Multiple Sclerosis Competence Centre, Dept. of } \\
\text { Neurology; } \\
\text { Myhr, Kjell; Haukeland Universisty Hospital, Neurology } \\
\text { Celius, Elisabeth; Nevrologisk avdeling, Oslo Universitetssykehus } \\
\text { Benjaminsen, Espen; Nordland Hospital, Neurology } \\
\text { Kampman, Margitta; University Hospital of North Norway, Neurology } \\
\text { Midgard, Rune; Molde Hospital, Department of Neurology } \\
\text { Vatne, Anita; Kristiansand Hospital, Neurology } \\
\text { Aarseth, Jan Harald; Haukeland University Hospital, The Norwegian } \\
\text { Multiple Sclerosis Registry and Biobank, Department of Neurology; } \\
\text { University of Bergen, KG Jebsen Centre for MS Research, Department of } \\
\text { Clinical Medicine } \\
\text { Rise, Trond; Universtity of Bergen, Department of Global Public Health } \\
\text { and Primary Care; Haukeland University Hospital, The Norwegian } \\
\text { Multiple Sclerosis Competence Center, Department of Neurology } \\
\text { Torkildsen, Øivind; Norwegian Multiple Sclerosis Competence Centre, } \\
\text { Department of Neurology }\end{array}$ \\
\hline Keywords: & Multiple sclerosis, cancer, siblings, prospective, cohort \\
\hline Abstract: & $\begin{array}{l}\text { Background } \\
\text { Risk of cancer in multiple sclerosis patients compared to their siblings is } \\
\text { unknown. } \\
\text { Objective } \\
\text { Prospectively investigate the risk of cancer among MS patients compared } \\
\text { to siblings without MS and to population controls. } \\
\text { Methods } \\
\text { We retrieved data on MS patients born } 1930 \text { - } 1979 \text { from the Norwegian } \\
\text { Multiple Sclerosis Registry, population studies, cancer diagnosis from the } \\
\text { Cancer Registry of Norway. We used adjusted Cox proportional hazard }\end{array}$ \\
\hline
\end{tabular}


regression to estimate cancer risk among 6883 MS patients, 8918 siblings without MS and 37,919 population controls.

Results

During 65 years follow-up, cancer risk among MS patients was higher than among population controls (Hazard Ratio $=1.14,95 \%$ CI $1.05-$ $1.23)$, in respiratory - $(H R=1.66,1.26-2.19)$, urinary - organs $(H R=1.51,1.12-2.04)$ and central nervous system $(H R=1.52,1.11-2$. 09). Siblings had higher risk of hematological cancers compared with MS patients $(\mathrm{HR}=1.82,1.21-2.73)$ and population controls $(\mathrm{HR}=1.72$, 1.36-2.18).

Conclusion

MS was associated with increased risk of cancer compared to population controls. Siblings had increased risk of hematological cancer. This indicates that MS and hematological cancer could share a common etiology.

\section{SCHOLARONE Manuscripts}




\title{
Risk of cancer among multiple sclerosis patients, siblings and population controls: a prospective cohort study
}

\author{
Nina Grytten, PhD ${ }^{1}$, Kjell-Morten Myhr, MD, PhD ${ }^{2,3}$, Elisabeth G. Celius, MD, PhD ${ }^{4,5}$ Espen \\ Benjaminsen ${ }^{6}$, Margitta Kampman ${ }^{7}$, Rune Midgard ${ }^{8,9}$, Anita Vatne ${ }^{10}$, Jan H. Aarseth ${ }^{11}$, Trond Riise \\ 1,12, Øivind Torkildsen 1, 2, 3
}

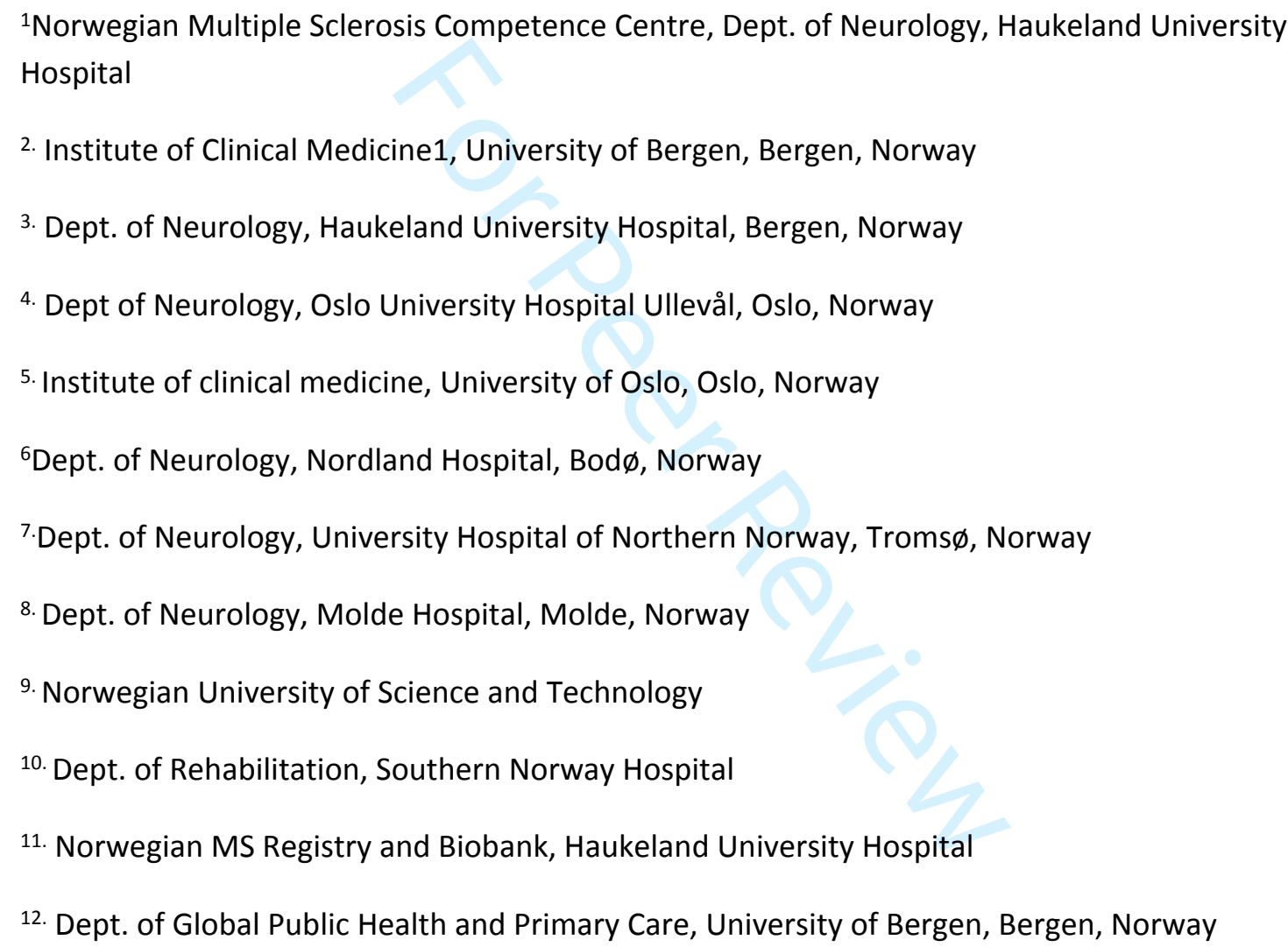
Neurology, Haukeland University Hospital. E- mail: nina.grytten@helse-bergen.no

Key words: Multiple sclerosis, risk, cancer, epidemiology

Twitter: Multiple sclerosis patients' siblings have increased risk of hematological cancers 


\section{Abstract \\ Background}

Risk of cancer in multiple sclerosis patients compared to their siblings is unknown.

\section{Objective}

Prospectively investigate the risk of cancer among MS patients compared to siblings without MS and to population controls.

\section{Methods}

We retrieved data on MS patients born 1930 - 1979 from the Norwegian Multiple Sclerosis Registry, population studies, cancer diagnosis from the Cancer Registry of Norway. We used adjusted Cox proportional hazard regression to estimate cancer risk among 6883 MS patients, 8918 siblings without MS and 37,919 population controls.

\section{Results}

During 65 years follow-up, cancer risk among MS patients was higher than among population controls $($ Hazard Ratio $=1.14,95 \%$ CI 1.05-1.23), in respiratory - $(\mathrm{HR}=1.66,1.26-2.19)$, urinary - organs $(\mathrm{HR}=1.51,1.12-2.04)$ and central nervous system $(\mathrm{HR}=1.52,1.11-2.09)$. Siblings had higher risk of hematological cancers compared with $\mathrm{MS}$ patients $(\mathrm{HR}=1.82,1.21-2.73)$ and population controls $(\mathrm{HR}=1.72,1.36-2.18)$

\section{Conclusion}

MS was associated with increased risk of cancer compared to population controls. Siblings had increased risk of hematological cancer. This indicates that MS and hematological cancer could share a common etiology. 


\section{Introduction}

Multiple sclerosis (MS) is an immune-mediated disease of the central nervous system (CNS). The immune system is involved in controlling and preventing cancer, and it is hypothesized that altered immune responses, inflammation and immunomodulating therapy could increase the risk of developing cancer $^{1}$. Cancer in MS might mirror immune system imbalances, and the chronic inflammation resulting from MS could cause MS patients to be more susceptible to cancer. ${ }^{2}$ Others have argued that the risk of cancer among MS patients is higher because of surveillance bias caused by frequent magnetic resonance imaging scans, which identifies CNS tumors at an earlier stage for MS patients. ${ }^{3}$ Immunotherapy for MS may potentially increase the risk of cancer among MS patients, as shown for treatment with chemotherapies ${ }^{4}$. Some studies have found either reduced overall risk of cancer $^{3,5,6}$ or no difference. ${ }^{7-9}$ Nevertheless, other studies have observed increased risk of developing malignancies in the digestive system and respiratory organs, ${ }^{5-7,10}$ male and female genital organs, skin, $^{3,5,11}$ breast, ${ }^{2,5,12-14}$ brain $^{3,15}$, urinary organs ${ }^{2,3,6}$ and lymphoma. ${ }^{3}$

These conflicting findings could result from heterogenity in study design and data sampling. With some notable exceptions, ${ }^{3,5}$ most studies on the risk of cancer in MS are based on administrative data, which are collected to inform on management issues rather than research purposes, ${ }^{6,16-18}$ or surveys and questionnaires. ${ }^{19,20}$ Only one previous study has compared cancer risk within family, reporting an increased cancer risk among fathers compared with their offspring with $\mathrm{MS}^{3}$

We therefore conducted a large population-based cohort study with data retrieved from national registries and published population studies to collect objective and reliable information on the risk of cancer among MS patients. The aim was to investigate the risk of cancer in MS prospectively. We compared MS patients with two control groups: controls from the general population of Norway and non-MS siblings of MS patients. We adjusted both groups for age, sex, area of residence and education, a marker of socioeconomic status. ${ }^{21}$ MS patients were compared with their siblings, since common genetics and exposure during childhood and adolescence might influence the disposition for malignant disease because of heritability, environmental factors or epigenetic interaction. We 
hypothesized that the chronic inflammation involved in MS could alter the risk of cancer among MS patients.

\section{Methods}

Study population and study design

The Norwegian Multiple Sclerosis Registry ${ }^{22}$ was the primary source for identifying patients in this study. The Registry, established in 2001, contains data for 8000 individuals with MS. In 2011, we conducted a sample of population-based epidemiological data on MS patients born between 1930 and 1979 in Norway retrieved from previously published population studies ${ }^{23,24}$ and included cases not identified in the registries at the date for data extraction, as described in the previously published studies using the same cohort. ${ }^{8,23,24}$ In addition, we included data from about 1200 patients with MS in a cohort enrolled in the Oslo Multiple Sclerosis Registry. ${ }^{25}$ We retrieved the place of birth, sex and data on all of patients' unaffected siblings and their year of birth from the Norwegian Population Registry (The Norwegian Tax Administration), established in 1964, for patients born from 1930 to 1979. The number of siblings ranged from 1 to 13 .

Patients had been diagnosed with MS according to the criteria of Poser et al. ${ }^{26}$ or McDonald. ${ }^{27} \mathrm{We}$ individually matched MS patients with five controls provided by Statistics Norway, adjusted for birth year, area of residence and sex.

We linked the complete cohort of cases $(n=6883)$, siblings $(n=9067)$ and population controls $(n=53,720)$ to the Cancer Registry of Norway, which was established in 1952. All cancer cases are required to be registered in the Cancer Registry of Norway, providing annual incidence data of cancer.

The Cancer Registry of Norway provided incidence data on diagnosis according to the International Classification of Diseases versions 7-10 (ICD-7-10). We retrieved the date of diagnosis for all MS patients, siblings and population controls until December 31, 2016. We obtained data on educational 
level for all cases from the National Education Database, which records all individually based data on education. Level of education was included in the model as a proxy for socioeconomic status.

To collect data on county of residence, the date for linking patients and controls by county of residence was set to their 15th birthday, to match for exposures related to residence when growing up. Data on residence were not available before the 1960 census from the Norwegian Population Statistics. We chose pragmatically the 15 th birthday as the index date to be set as early in the preclinical course as possible while still being able to implement registered residence for the majority in the sample.

A total of 4495 MS patients had one or more siblings, and we compared their individual risk of cancer with that of their own siblings. Thus, we excluded 2390 patients with no sibling from the analysis when comparing the risk of cancer among MS patients and siblings. However, we included the total cohort of MS patients $(n=6883)$ in the analysis of risk of cancer among MS patients compared with the controls from the general population. Table 1 describes the cohort, including the two MS patient categories. 
Statistical analysis

We used Cox proportional hazard regression to estimate the risk of cancer among MS patients, their siblings and the controls. We report hazard ratio (HR) with $95 \%$ confidence intervals $(\mathrm{CI})$ as an estimate of the association between having MS and cancer risk. Follow-up was from the time the Cancer Registry of Norway was established in 1952 or subsequently from birth or immigration. We followed up patients, the siblings and the population controls until date of diagnosis of any cancer, death or emigration or the end of follow-up on December 31, 2016. Individuals not developing cancer were censored at date of emigration, death or end of follow-up, whichever occurred first. The results were reported for the risk of first primary cancer. When analyzing subgroups of cancers, individuals who developed another type of cancer were censored at this time. We included sex, age, area (county) of residence and attained educational level as covariates in the Cox-model. We categorized level of education into primary level (10 years or less), secondary level (11-13 years), undergraduate level (14-17 years) and graduate level (18 years or more). When analyzing the cancer risk between MSpatients and their siblings we adjusted for the dependency within each group of siblings by running a Cox-regression with robust standard errors using the cluster option in STATA.

We generated categories of cancer based on data from the Cancer Registry of Norway originally based on ICD-7-10 into: oral cavity and larynx $(\mathrm{C} 0-\mathrm{C} 14)$, digestive system $(\mathrm{C} 15-\mathrm{C} 26)$, respiratory organs (C30-C39), bones and joints (C40-C42, C45-C49), skin (C43-C44), breast (C50), female genital organs (C51-C58), male genital organs (C60-C63), urinary organs (C64-C68), eye and adnexa (C69), central nervous system (CNS) (C70-C72), including meninges (C70); thyroid and other endocrine glands (C73-C75), unspecified (C76, C80), "hematological cancers" including lymphoma, myeloma, hematopoietic or lymphatic" (C81-C96, D45-D46).

We estimated the risks of overall cancer and organ or system-specific cancer diagnosis and performed separate analyses for men and women. We also performed separate stratified analyses for time periods including birth before and after 1958, the median birth year for participants. This enabled us to 
evaluate a possible risk associated with immunomodulatory therapy, which became available in the mid-1990s, and specifically for participants born after 1958 .

We performed the statistical analysis in Stata Statistical Software: Release 15 (StataCorp, College Station, TX, USA), and IBM SPSS Statistics 24.

\section{Ethical approval}

The Western Norway Regional Committee for Medical and Health Research Ethics approved the study (REK Vest 2016/300).

\section{Results}

We identified 6883 MS patients, 37,919 population controls, 4493 MS patients with siblings and 8918 siblings altogether (Table 1). A total of 4597 MS patients (67\%), 25,265 general population controls (67\%), 2980 MS patients with siblings (66\%) and 4256 of siblings of MS patients (48\%) were women.

A diagnosis of cancer was recorded for $11.2 \%$ of the total MS population $(n=774), 10.6 \%$ of the population controls $(n=4017), 8.1 \%(n=366)$ in the subpopulation of MS patients with siblings and $9.3 \%(n=830)$ of the siblings of those MS patients.

Low educational level was associated with increased risk of cancer in the total population (HR lowest versus highest level) of 1.32 (95\% CI: 1.25-1.40), and there was no difference in the estimates between the groups. We therefore adjusted all Cox regression analysis with attained educational level, since previous studies have reported the inverse association between risk of cancer and education. ${ }^{28}$

\section{Risk of cancer among MS patients compared with population controls}


The overall risk of cancer was higher among MS patients than among population controls $(\mathrm{HR}=1.14$, 95\% CI 1.05-1.23) (Table 2). Women with MS had significant excess risk of cancer (HR $=1.18,95 \%$ CI 1.07-1.29), but not men $(\mathrm{HR}=1.05,95 \%$ CI $0.92-1.21)$.

Organ-specific analysis revealed significant excess of cancer among MS patients compared with the population controls in the respiratory organs $(\mathrm{HR}=1.66,95 \% \mathrm{CI}: 1.26-2.19)$, urinary organs $(\mathrm{HR}=$ 1.51, 95\% CI: $1.12-2.04)$ and CNS (HR $=1.52,95 \% \mathrm{CI}: 1.11-2.09)$. In the CNS, MS patients had specifically increased risk of cancer of the meninges ( $\mathrm{HR}=1.95,95 \% \mathrm{CI}: 1.26-3.01)$. Median age among MS patients for diagnosis of cancer in the meninges was 54 years, compared with 56 years among controls.

We repeated the analysis after excluding cancer diagnosis in the respiratory organs, urinary organs and CNS. The results revealed similar risk of cancer among MS patients and population controls $(\mathrm{HR}=$ $1.05,95 \%$ CI: $0.97-1.15)$, indicating that the increased risk of cancer was mainly attributable to cancer in these organ systems.

There was no significant difference in the risk of overall cancer between the cohorts born before and after $1958(\mathrm{HR}=1.17,95 \%$ CI: $1.08-1.28$ vs. HR: $1.14,95 \%$ CI: $0.96-1.35)$, test of interaction, $\mathrm{p}=$ 0.75 .

\section{Risk of cancer among MS patients compared with their siblings}

The MS patients had a non-significant lower overall risk of cancer compared with their siblings without MS (HR $=0.92,95 \%$ CI: $0.83-1.03)$, similar for women $(\mathrm{HR}=0.94,95 \% \mathrm{CI}: 0.82-1.09)$ and men $(\mathrm{HR}=0.89,95 \% \mathrm{CI}: 0.75-1.07)$ (Table 3$)$. Organ-specific analysis revealed a significantly lower risk of hematological cancers among MS patients compared with their siblings without MS (HR = 0.55, 95\% CI: $0.37-0.82$ ). There was a difference, although not significant in the risk of overall cancer between the cohorts born before or after 1958 ( $\mathrm{HR}=1.95,95 \% \mathrm{CI}: 0.78-1.15$ vs. $\mathrm{HR}=0.91,95 \% \mathrm{CI}$ : $0.80-1.05)$, test of interaction, $p=0.47$. The results of the Cox regression analysis revealed the same 
trend both with the full cohort of MS patients $(n=6883)$ and the MS patients who had siblings $(n=$ 4493).

\section{Increased risk of hematological cancer among siblings of MS patients}

We found an increased risk of hematological cancers among the siblings compared with MS patients (HR: 1.82, 1.21-2.73), especially an increased risk of lymphoma (HR: 1.75, 95\% CI: 0.99-3.12) (Fig. 1a).

We also found increased risk of hematological cancers among the siblings compared with population controls (1.72, 95\% CI: 1.36-2.18). Specifically, lymphoma (HR: 1.49, 95\% CI: 1.07-2.09), myeloma (HR: 2.04, 95\% CI: 1.25-4.64) and leukemia (HR: 1.62, 95\% CI: 1.01-2.62) were significantly increased among the siblings of MS patients compared with population controls (Fig. 1b).

Also, the overall risk of cancer $(\mathrm{HR}=1.21,95 \% \mathrm{CI} 1.12-1.31)$ and cancer in the respiratory organs (HR: 1.40, 95\% CI: 1.04-1.89) was higher among siblings of MS patients than among population controls (Table 4).

\section{Discussion}

We have performed a prospective population-based cohort study with an average of 65 years of follow-up of MS patients, their siblings and population controls. We found an overall $14 \%$ increased risk of cancer among MS patients compared with population controls, especially in respiratory organs, urinary organs and the CNS. However, although the overall cancer risk is not significant increased among men, unlike women, the results showed that male MS patients had the same increased risk as female MS patients for cancer in CNS meninges, respiratory- and urinary organs. The overall cancer risk for men was markedly influenced by the low risk for male genital cancer (prostate). 
In the CNS, meningioma was especially increased among MS patients compared with population controls. However, MS patients did not have an increased risk of cancer compared with their siblings, and siblings had a markedly increased risk of hematological cancers, especially lymphomas. Siblings of MS patients also had a higher incidence of myeloma and leukemia than population controls.

Our result showing that MS patients have an increased risk of cancer in the CNS, mainly meningioma, is consistent with previous studies. ${ }^{3,4} \mathrm{We}$ also found that MS patients were diagnosed two years earlier than the controls with cancer in the meninges. This could partly support the hypothesis of surveillance bias and the early identification of meningioma related to frequent magnetic resonance scanning of MS patients, which increases the probability of identifying brain tumors, including meningioma, among MS patients. Other neoplasms in the CNS would eventually manifest during the course of disease, also among people without MS. Consequently, the increased incidence of meningioma, possibly benign, among MS patients can be attributed to frequent surveillance of the CNS. Future studies could adjust for amount of health care utilization to further exploring the role of MRI and surveillance in identifying early CNS cancer. However, we excluded benign neoplasms of cerebral meninges (ICD-10: D32) from our analysis, indicating that the increased risk of cancer in the CNS cannot be fully explained by surveillance bias. Meningioma could be caused by chronic inflammation, and the increased risk of cancer in the CNS, including meningioma, could result from MS-specific disease activity: the inflammatory process and the immune response in $\mathrm{CNS}^{29}$.

The observed increased overall risk of cancer among our MS patients differs from previous studies reporting that MS patients have lower overall risk than the general population. ${ }^{3,5,6,11}$ Several factors might explain this difference. First, diagnostic neglect and underestimation of cancer incidence could explain some of the lower risk of cancer reported previously. ${ }^{6}$ Second, lower cancer incidence among the population controls could explain the increased risk of cancer among MS patients in Norway. The cancer incidence in 2018 is 337,8/100,000 for both sexes in Norway, but lower incidences have been reported in Sweden ${ }^{30}$. Third, excessive smoking among MS patients compared with the general population in Norway could cause increased risk of cancer. ${ }^{31}$ We observed concordant 
excess risk in the urinary organs and respiratory organs, both types of cancer strongly associated with smoking ${ }^{14}$. However, we cannot rule out bladder dysfunction and urinary infections that might cause chronic irritation and hence urinary tract cancer among MS patients. ${ }^{3}$ Finally, study design may influence the result, and the end of study is a plausible reason for the increased risk of cancer among MS patients in Norway: 2017 in Norway, 2005 in Sweden and 1995 in Denmark. Immunomodulatory therapy (IMT) in MS might potentially increase the risk of cancer, ${ }^{4,32}$ and such treatment has been available in Norway, although not extensively prescribed, since 1996-1997. Hence, there could be more patients treated with IMT in our cohort, possibly explaining some of the higher risk of cancer among MS patients in Norway. However, we have no exact data on the use of IMT in this sample. Although we found no change in risk of cancer associated with MS in the younger cohort, we cannot rule out the potential risk of cancer associated with these therapies, since longer follow-up time from drug exposure is probably needed to detect a potential risk of cancer.

Both MS patients and their siblings had overall increased risk of cancer compared with the population controls. This familial risk of cancer supports the hypothesis of genetic risk and common environmental conditions and lifestyles. However, compared with both MS patients and population controls we observed siblings of MS patients to be more susceptible to hematological cancers. Previous studies of a familial clustering of hematological cancers support a hypothesis of shared etiology in MS and hematological cancers, reported as Hodgkin lymphoma among the first-degree relatives of MS patients ${ }^{33}$ and among the fathers of MS patients. ${ }^{3}$ These observations of familial clustering of MS and hematological cancers are also consistent with the hypothesis launched in 1970 suggesting shared either genetic susceptibility, environmental factors or both. ${ }^{34}$ Genetic studies have indicated a common mechanism between Hodgkin's lymphoma and MS, suggesting genetics and epigenetics as common risk factors for both diseases. ${ }^{29}$ Exposure to Epstein-Barr virus in a family setting could be a possible environmental factor, resulting in either MS or hematological cancer among the siblings of MS patients, since the same epigenetic factors probably regulate both diseases. ${ }^{29}$ 
MS patients have an average of 8 years shorter life expectancy than population controls..$^{35,36}$ We used the Cox method, and thus the potential bias related to survival or immortal time is unlikely to occur, since the Cox model calculates the age-specific risks and time-dependent hazard ratios.

Including two independent control groups without MS strengthens the validity of the study. The observations of increased risk of cancer among MS patients compared with population controls, no increased risk of cancer among MS patients compared with their siblings and the higher risk of hematological cancer among the siblings all support the hypothesis of a shared genetic risk for MS and certain cancers.

The use of national registries for reliable information on exposure and diagnosis at the population level is a strength giving this study validity. The Cancer Registry has an almost complete database of all incident cancer cases, and the diagnostic accuracy is reliable, ${ }^{37}$ reducing the risk of potential information bias.

A potential limitation of our study was the lack of behavioral data and lifestyle information such as smoking habits. However, the data enabled us to adjust for level of education (as a proxy for socioeconomic status), in addition to sex, age and area of residence. Finally, we did not adjust for multiple testing when estimating the subgroup cancer risks and these results should therefore be interpreted with caution.

In conclusion, MS patients had an increased risk of cancer in the respiratory organs, the urinary organs and in the CNS compared with the population controls that might be caused by excessive smoking and surveillance bias, although an increased incidence of meningioma indicates that chronic inflammation could also contribute. Siblings of MS patients had an increased incidence of hematological cancers compared with both MS patients and the population controls. The increased risk of hematological cancers, verified by using two control groups, suggests that MS and hematological cancer could share a common etiology that can be important for future treatment of MS and prevention of both diseases. 


\section{Declaration of Conflicting Interests}

This study was funded by the Western Norway Regional Health Authority, and supported by T Hauge's legacy.

Dr. Grytten reports no disclosures. Dr. Riise reports no dislcosures. Dr. Aarseth reports no disclosures. Dr. Vatne reports no disclosures. Dr. Midgard reports no disclosures. Dr. Benjaminsen reports no disclosures. Dr. Myhr reports grants and personal fees from Biogen Idec, grants and personal fees from Novartis, personal fees from Genzyme, personal fees from Roche, personal fees from Almirall, personal fees from Merck, personal fees and non-financial support from Teva, outside the submitted work; Dr. Celius reports grants and personal fees from Sanofi Genzyme, personal fees from Biogen, personal fees from Teva, personal fees from Merck, personal fees from Roche, personal fees from Almirall, grants and personal fees from Novartis, outside the submitted work; Dr.Torkildsen reports personal fees from Biogen, personal fees from Merck, personal fees from Sanofi, personal fees from Roche, personal fees from Teva, outside the submitted work.

\section{Appendix}

The study has used data from the Cause of death Registry and the Cancer Registry of Norway. The authors are solely responsible for interpreting and reporting these data, and no endorsement by the Cause of death Registry or the Cancer Registry of Norway is intended or should be inferred. 


\section{Uncategorized References}

1. Tan TT and Coussens LM. Humoral immunity, inflammation and cancer. Current opinion in immunology. 2007; 19: 209-16.

2. Ajdacic-Gross V, Rodgers S, Aleksandrowicz A, et al. Cancer co-occurrence patterns in Parkinson's disease and multiple sclerosis-Do they mirror immune system imbalances? Cancer epidemiology. 2016; 44: 167-73.

3. Bahmanyar S, Montgomery SM, Hillert J, Ekbom A and Olsson T. Cancer risk among patients with multiple sclerosis and their parents. Neurology. 2009; 72: 1170-7.

4. Buttmann M, Seuffert L, Mader U and Toyka KV. Malignancies after mitoxantrone for multiple sclerosis: A retrospective cohort study. Neurology. 2016; 86: 2203-7.

5. Nielsen NM, Rostgaard K, Rasmussen S, et al. Cancer risk among patients with multiple sclerosis: a population-based register study. International journal of cancer. 2006; 118: 979-84.

6. Kingwell E, Bajdik C, Phillips N, et al. Cancer risk in multiple sclerosis: findings from British Columbia, Canada. Brain : a journal of neurology. 2012; 135: 2973-9.

7. Sumelahti ML, Pukkala E and Hakama M. Cancer incidence in multiple sclerosis: a 35-year follow-up. Neuroepidemiology. 2004; 23: 224-7.

8. Bjornevik K, Riise T, Benjaminsen $\mathrm{E}$, et al. Level of education and multiple sclerosis risk over a 50-year period: Registry-based sibling study. Multiple sclerosis. 2017; 23: 213-9.

9. Midgard R, Glattre E, Gronning M, Riise T, Edland A and Nyland H. Multiple sclerosis and cancer in Norway. A retrospective cohort study. Acta neurologica Scandinavica. 1996; 93: 411-5. 10. Johannesson M, Askling J, Montgomery SM, Ekbom A and Bahmanyar S. Cancer risk among patients with cystic fibrosis and their first-degree relatives. International journal of cancer. 2009; 125 : 2953-6.

11. Goldacre MJ, Seagroatt V, Yeates D and Acheson ED. Skin cancer in people with multiple sclerosis: a record linkage study. Journal of epidemiology and community health. 2004; 58: 142-4. 12. Hajiebrahimi M, Montgomery S, Burkill S and Bahmanyar S. Risk of Premenopausal and Postmenopausal Breast Cancer among Multiple Sclerosis Patients. PloS one. 2016; 11: e0165027. 13. Koch-Henriksen N. The Danish Multiple Sclerosis Registry: a 50-year follow-up. Multiple sclerosis. 1999; 5: 293-6.

14. Sun LM, Lin CL, Chung CJ, Liang JA, Sung FC and Kao CH. Increased breast cancer risk for patients with multiple sclerosis: a nationwide population-based cohort study. European journal of neurology. 2014; 21: 238-44.

15. Fois AF, Wotton CJ, Yeates D, Turner MR and Goldacre MJ. Cancer in patients with motor neuron disease, multiple sclerosis and Parkinson's disease: record linkage studies. Journal of neurology, neurosurgery, and psychiatry. 2010; 81: 215-21.

16. Kingwell E, Evans C, Zhu F, Oger J, Hashimoto $S$ and Tremlett H. Assessment of cancer risk with beta-interferon treatment for multiple sclerosis. Journal of neurology, neurosurgery, and psychiatry. 2014; 85: 1096-102.

17. Kingwell $\mathrm{E}$ and Tremlett $\mathrm{H}$. Interferons and multiple sclerosis: is it plausible that beta-IFN treatment could influence the risk of cancer among MS patients? Expert review of neurotherapeutics. 2009; 9: 1263-5.

18. Marrie RA, Reider N, Cohen J, et al. A systematic review of the incidence and prevalence of cancer in multiple sclerosis. Multiple sclerosis. 2015; 21: 294-304.

19. Gaindh D, Kavak KS, Teter B, et al. Decreased risk of cancer in multiple sclerosis patients and analysis of the effect of disease modifying therapies on cancer risk. Journal of the neurological sciences. 2016; 370: 13-7.

20. Moisset $\mathrm{X}$, Perie $\mathrm{M}$, Pereira $\mathrm{B}$, et al. Decreased prevalence of cancer in patients with multiple sclerosis: A case-control study. PloS one. 2017; 12: e0188120.

21. Shavers VL. Measurement of socioeconomic status in health disparities research. J Natl Med Assoc. 2007; 99: 1013-23. 
22. Myhr KM, Grytten N, Torkildsen O, Wergeland S, Bo L and Aarseth JH. The Norwegian Multiple Sclerosis Registry and Biobank. Acta neurologica Scandinavica. 2015; 132: 24-8.

23. Kampman MT, Aarseth JH, Grytten N, et al. Sex ratio of multiple sclerosis in persons born from 1930 to 1979 and its relation to latitude in Norway. Journal of neurology. 2013; 260: 1481-8.

24. Grytten N, Torkildsen $\mathrm{O}$, Aarseth JH, et al. Month of birth as a latitude-dependent risk factor for multiple sclerosis in Norway. Multiple sclerosis. 2013; 19: 1028-34.

25. Celius EG and Smestad C. Change in sex ratio, disease course and age at diagnosis in Oslo MS patients through seven decades. Acta Neurol Scand Suppl. 2009: 27-9.

26. Poser CM, Paty DW, Scheinberg L, et al. New diagnostic criteria for multiple sclerosis: guidelines for research protocols. Annals of neurology. 1983; 13: 227-31.

27. Polman CH, Reingold SC, Edan G, et al. Diagnostic criteria for multiple sclerosis: 2005 revisions to the "McDonald Criteria". Annals of neurology. 2005; 58: 840-6.

28. Mouw T, Koster A, Wright ME, et al. Education and risk of cancer in a large cohort of men and women in the United States. PloS one. 2008; 3: e3639.

29. Frampton $M$, da Silva Filho $\mathrm{Ml}$, Broderick $\mathrm{P}$, et al. Variation at 3p24.1 and $6 q 23.3$ influences the risk of Hodgkin's lymphoma. Nat Commun. 2013; 4: 2549.

30. http://gco.iarc.fr/today/home. Cancer today. WHO. International Agency for Research on Cancer 2019, 2018.

31. Riise T, Nortvedt MW and Ascherio A. Smoking is a risk factor for multiple sclerosis. Neurology. 2003; 61: 1122-4.

32. Confavreux C, Saddier P, Grimaud J, Moreau T, Adeleine P and Aimard G. Risk of cancer from azathioprine therapy in multiple sclerosis: a case-control study. Neurology. 1996; 46: 1607-12.

33. Hjalgrim $\mathrm{H}$, Rasmussen $\mathrm{S}$, Rostgaard $\mathrm{K}$, et al. Familial clustering of Hodgkin lymphoma and multiple sclerosis. J Natl Cancer Inst. 2004; 96: 780-4.

34. Newell GR. Etiology of multiple sclerosis and Hodgkin's disease. Am J Epidemiol. 1970; 91: 119-22.

35. Lunde HMB, Assmus J, Myhr KM, Bo L and Grytten N. Survival and cause of death in multiple sclerosis: a 60-year longitudinal population study. Journal of neurology, neurosurgery, and psychiatry. 2017; 88: 621-5.

36. Thormann A, Sorensen PS, Koch-Henriksen N, Laursen B and Magyari M. Comorbidity in multiple sclerosis is associated with diagnostic delays and increased mortality. Neurology. 2017.

37. Larsen IK, Smastuen M, Johannesen TB, et al. Data quality at the Cancer Registry of Norway: an overview of comparability, completeness, validity and timeliness. European journal of cancer.

2009; 45: 1218-31. 


\begin{tabular}{|c|c|c|c|c|}
\hline & $\begin{array}{l}\text { Total } \\
n(\%)\end{array}$ & $\begin{array}{l}\text { Total } \\
n(\%)\end{array}$ & $\begin{array}{l}\text { Total } \\
n(\%)\end{array}$ & $\begin{array}{l}\text { Total } \\
n(\%)\end{array}$ \\
\hline \multicolumn{5}{|l|}{ Sex } \\
\hline Female & $4597(66.8)$ & $25,265(66.6)$ & $2980(66.4)$ & $4256(47.7)$ \\
\hline Male & $2286(33.2)$ & $12,654(33.4)$ & $1513(33.6)$ & $4662(52.3)$ \\
\hline Age (years), median, SD & $61.0(11.5)$ & $61.0(11.6)$ & $57.0(9.3)$ & $57.0(9.9)$ \\
\hline Year of birth, median, SD & $1956(11.5)$ & $1956(11.6)$ & $1960(9.3)$ & $1959(9.9)$ \\
\hline \multicolumn{5}{|l|}{ Education } \\
\hline Graduate level & $443(6.4)$ & $2707(7.1)$ & $333(7.4)$ & $634(7.0)$ \\
\hline $\begin{array}{l}\text { Age at cancer diagnosis } \\
\text { (years), median (SD) } \\
\text { Cancer: malignant neoplasm } \\
\text { of: }\end{array}$ & $57.35(11.9)$ & $58.24(13.7)$ & $52.5(11.7)$ & $52.47(13.8)$ \\
\hline Overall & $774(11.2)$ & $4017(10.6)$ & $366(8.1)$ & $830(9.3)$ \\
\hline Brain and nervous system & $49(6.3)$ & $190(4.7)$ & $27(7.4)$ & $51(6.1)$ \\
\hline Meninges & $27(3.5)$ & $81(2.0)$ & $14(3.8)$ & $14(1.7)$ \\
\hline Breast & $160(20.7)$ & $837(20.8)$ & $78(21.3)$ & $127(15.3)$ \\
\hline Skin & $74(9.6)$ & 469 (11.7) & $49(13.4)$ & $97(11.7)$ \\
\hline
\end{tabular}


Eye and adnexa

Endocrine glands

Hematological cancers;

Lymphoid, hematopoietic

and related tissue

Oral cavity and larynx

Respiratory organs

Unknown
$0(0)$

$25(3.2)$

$48(6.2)$

$14(0.3)$

$104(2.6)$

$298(7.4)$

$0(0.0)$

$12(3.3)$

$24(6.6)$

$3(0.4)$

$26(3.1)$

98 (11.8)

$9(1.2)$

$65(8.4)$

$10(1.3)$
$59(1.5)$

$231(5.8)$

$22(0.5)$
$6(1.6)$

$20(5.5)$

$1(0.03)$
$7(0.8)$

$58(7.0)$ 
Table 2. Risk of primary cancer among MS patients compared with controls from the general population without MS

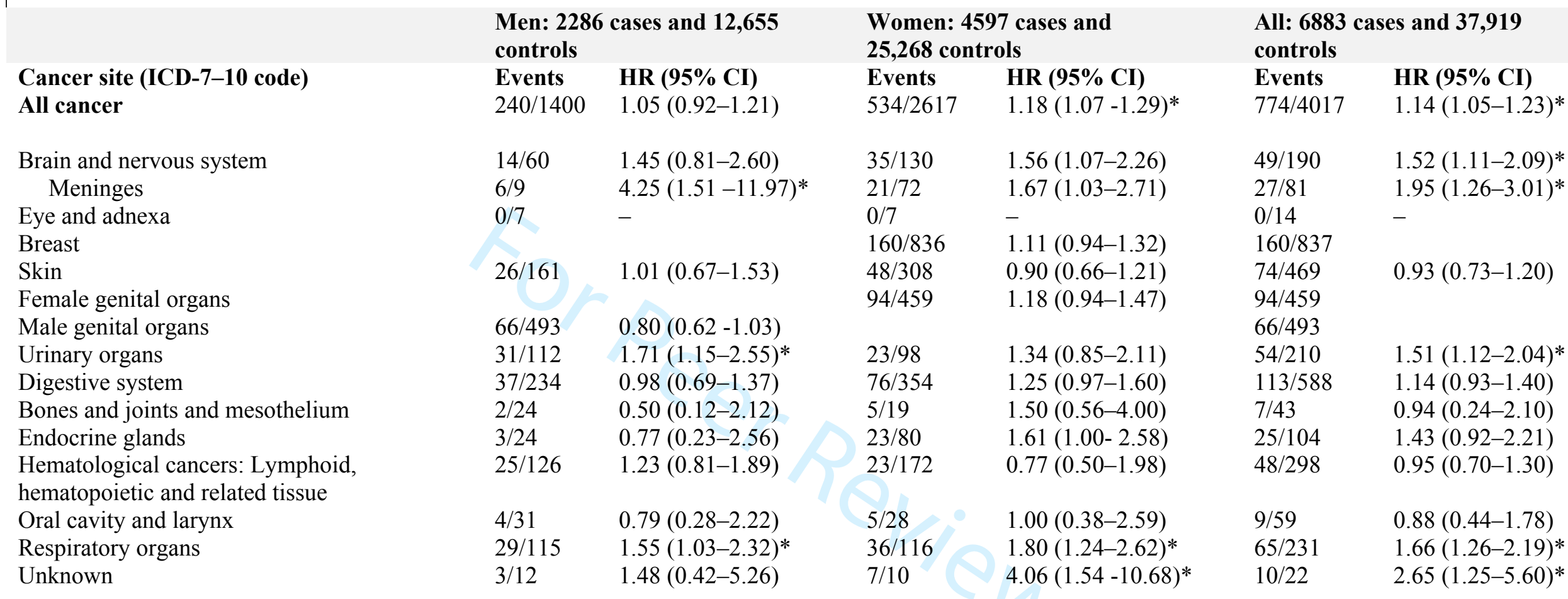

The model was adjusted for age, sex, residence and attained educational level.

$\mathrm{HR}=$ hazard ratio. $\mathrm{CI}=$ confidence interval.

$* \mathrm{P} \leq 0.05$ 
Table 3. Risk of primary cancer among MS patients compared with their siblings without MS

\begin{tabular}{|c|c|c|c|c|c|c|}
\hline \multirow{3}{*}{$\begin{array}{l}\text { Cancer site (ICD-7-10 code) } \\
\text { All cancer }\end{array}$} & \multicolumn{2}{|c|}{$\begin{array}{l}\text { Men: } 1513 \text { cases and } 4662 \\
\text { siblings }\end{array}$} & \multicolumn{2}{|c|}{$\begin{array}{l}\text { Women: } 2980 \text { cases and } 4256 \\
\text { siblings }\end{array}$} & \multicolumn{2}{|c|}{ All: 4493 cases and 8918 siblings } \\
\hline & Events & HR (95\% CI) & Events & HR $(95 \%$ CI $)$ & Events & HR (95\% CI) \\
\hline & $110 / 411$ & $0.89(0.75-1.07)$ & $256 / 419$ & $0.94(0.82-1.09)$ & $366 / 830$ & $0.92(0.83-1.03)$ \\
\hline Brain and nervous system & $8 / 28$ & $0.87(0.42-1.80)$ & $19 / 23$ & $1.15(0.72-2.25)$ & $27 / 51$ & $1.10(0.71-1.69)$ \\
\hline Meninges & $3 / 4$ & $2.33(0.59-9.16)$ & $11 / 10$ & $1.87(0.84-4.13)$ & $14 / 14$ & $1.98(0.99-3.99)$ \\
\hline Eye and adnexa & $0 / 3$ & 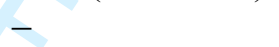 & $0 / 0$ & - & $0 / 3$ & - \\
\hline Breast & & & $78 / 127$ & $0.95(0.72-1.23)$ & $78 / 127$ & \\
\hline Skin & $14 / 48$ & $0.94(0.56-1.60)$ & $35 / 49$ & $0.97(0.64-1.47)$ & 49/97 & $0.97(0.70-1.27)$ \\
\hline Female genital organs & & & $43 / 76$ & $0.89(0.64,1,27)$ & $43 / 76$ & \\
\hline Male genital organs & $29 / 109$ & $0.93(0.66-1.31)$ & & & 29/109 & \\
\hline Urinary organs & $12 / 32$ & $1.36(0.78-2.37)$ & $10 / 11$ & $1.38(0.62-3.07)$ & $22 / 43$ & $1.37(0.87-2.17)$ \\
\hline Digestive system & $21 / 72$ & $0.68(0.56-1.37)$ & $30 / 40$ & $1.20(0.78-1.85)$ & $51 / 112$ & $1.02(0.75-1.39)$ \\
\hline $\begin{array}{l}\text { Bones and joints and } \\
\text { mesothelium }\end{array}$ & $1 / 10$ & $0.24(0.03-1.82)$ & $3 / 5$ & $0.73(0.17-3.03)$ & $4 / 15$ & $0.46(0.16-1.36)$ \\
\hline Endocrine glands & $2 / 9$ & $0.66(0.14-3.08)$ & $10 / 17$ & $0.84(0.39-1.84)$ & $12 / 26$ & $0.80(0.40-1.60)$ \\
\hline $\begin{array}{l}\text { Hematological cancers: } \\
\text { Lymphoid, hematopoietic and } \\
\text { related tissue }\end{array}$ & $11 / 59$ & $0.56(0.32-1.01)$ & $13 / 39$ & $0.53(0.31-0.99)^{*}$ & $24 / 98$ & $0.55(0.37-0.82)^{*}$ \\
\hline Oral cavity and larynx & $2 / 5$ & $1.36(0.32-5.88)$ & $4 / 2$ & $2.30(0.41-12.86)$ & $6 / 7$ & $1.71(0.59-4.93)$ \\
\hline Respiratory organs & $10 / 33$ & $0.87(0.56-1.37)$ & $10 / 25$ & $1.20(0.78-1.85)$ & $20 / 58$ & $1.02(0.75-1.39)$ \\
\hline Unknown & $0 / 3$ & - & $1 / 5$ & $0.47(0.09-2.45)$ & $2 / 8$ & $0.54(0.39-7.70)$ \\
\hline
\end{tabular}

The model was adjusted for age, sex, residence and attained educational level.

$\mathrm{HR}=$ hazard ratio. $\mathrm{CI}=$ confidence interval .

$* \mathrm{P} \leq 0.05$ 
Table 4. Risk of primary cancer among siblings of MS patients without MS compared with controls from the general population

\begin{tabular}{|c|c|c|c|c|c|c|}
\hline \multirow{3}{*}{$\begin{array}{l}\text { Cancer site (ICD-7-10 code) } \\
\text { All cancer }\end{array}$} & \multicolumn{2}{|c|}{$\begin{array}{l}\text { Men: } 4662 \text { cases and 12,654 } \\
\text { controls }\end{array}$} & \multicolumn{2}{|c|}{$\begin{array}{l}\text { Women: } 4256 \text { cases and } 25,265 \\
\text { controls }\end{array}$} & \multicolumn{2}{|c|}{$\begin{array}{l}\text { All: } 8918 \text { cases and } 37,919 \\
\text { controls }\end{array}$} \\
\hline & Events & HR $(95 \%$ CI) & Events & HR $(95 \%$ CI) & Events & HR (95\% CI) \\
\hline & $411 / 1400$ & $1.22(1.09-1.37)^{*}$ & $419 / 2617$ & $1.25(1.12-1.39)^{*}$ & $830 / 4017$ & $1.21(1.12-1.31)^{*}$ \\
\hline Brain and nervous system & $28 / 60$ & $1.38(0.88-2.19)$ & $23 / 130$ & $1.16(0.74-1.81)$ & $51 / 190$ & $1.26(0.92-1.73)$ \\
\hline Meninges & $4 / 9$ & $1.71(0.51-5.81)$ & $10 / 72$ & $1.02(0.52-1.99)$ & $14 / 81$ & $1.13(0.63-2.01)$ \\
\hline Eye and adnexa & $3 / 7$ & $1.19(0.30-4.71)$ & $0 / 0$ & - & $3 / 14$ & $0.85(0.24-3.05)$ \\
\hline Breast & & & $127 / 837$ & $1.17(0.97-1.42)$ & $127 / 837$ & \\
\hline Skin & $48 / 161$ & $1.24(0.89-1.74)$ & $49 / 308$ & $1.27(0.94-1.73)$ & $97 / 469$ & $1.24(0.99-1.55)$ \\
\hline Female genital organs & & & $76 / 459$ & $1.24(0.97-1.58)$ & $76 / 459$ & \\
\hline Male genital organs & $109 / 493$ & $1.01(0.81-1.25)$ & & & $109 / 493$ & \\
\hline Urinary organs & $32 / 112$ & $1.42(0.94-2.13)$ & $11 / 98$ & $0.94(0.50-1.77)$ & $43 / 210$ & $1.20(0.85-1.70)$ \\
\hline Digestive system & $72 / 234$ & $1.38(1.05-1.81)^{*}$ & $40 / 354$ & $1.04(0.75-1.46)$ & $112 / 588$ & $1.22(0.99-1.50)$ \\
\hline Bones and joints and mesothelium & $10 / 24$ & $1.25(0.59-2.65)$ & $5 / 19$ & $2.07(0.75-5.68)$ & $15 / 43$ & $1.50(0.82-2.77)$ \\
\hline Endocrine glands & $9 / 24$ & $1.25(0.57-2.74)$ & $17 / 80$ & $1.39(0.82-2.35)$ & $26 / 104$ & $1.31(0.86-2.01)$ \\
\hline $\begin{array}{l}\text { Hematological cancers: Lymphoid, } \\
\text { hematopoietic and related tissue }\end{array}$ & $59 / 126$ & $1.74(1.27-2.40)^{*}$ & $39 / 172$ & $1.73(1.21-2.47)^{*}$ & $98 / 298$ & $1.72(1.36-2.18)^{*}$ \\
\hline Oral cavity and larynx & $2 / 31$ & $0.60(0.23-1.58)$ & $2 / 28$ & $0.60(0.14-2.55)$ & $7 / 59$ & $0.60(0.27-1.33)$ \\
\hline Respiratory organs & $33 / 115$ & $1.19(0.80-1.78)$ & $25 / 116$ & $1.82(1.17-2.84)^{*}$ & $58 / 231$ & $1.40(1.04-1.89) *$ \\
\hline Unknown & $3 / 12$ & $0.80(0.22-2.90)$ & $5 / 10$ & $4.19(1.37-12.81) *$ & $8 / 22$ & $1.81(0.66-4.03)$ \\
\hline
\end{tabular}

The model was adjusted for age, sex, residence and attained educational level.

$\mathrm{HR}=$ hazard ratio. $\mathrm{CI}=$ confidence interval.

$* \mathrm{P} \leq 0.05$ 


\section{Figure legends}

1. Fig 1a. Hazard ratio (HR) and $95 \%$ confidence intervals (CI) for the association between hematological cancer of siblings of MS patients $(n=8918)$ and MS patients $(n=4493)$ in cluster analysis

2. Fig. 1b. Hazard ratio (HR) and $95 \%$ confidence intervals (CI) for the association between hematological cancer among the siblings of MS patients $(n=8918)$ and population controls $(n=37,919)$ 
Fig 1a. Hazard ratio (HR) and 95\% confidence intervals (CI) for the association between hematological cancer of siblings of MS patients $(n=8918)$ and MS patients $(n=4493)$ in cluster analysis

$338 \times 190 \mathrm{~mm}(300 \times 300 \mathrm{DPI})$ 


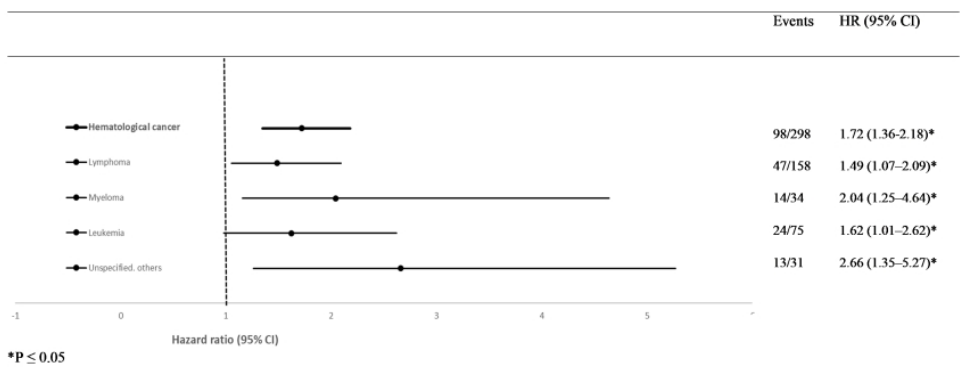

Fig. 1b. Hazard ratio (HR) and 95\% confidence intervals (CI) for the association between hematological cancer among the siblings of MS patients $(n=8918)$ and population controls $(n=37,919)$

$338 \times 190 \mathrm{~mm}(300 \times 300$ DPI $)$ 